\title{
Development and Validation of Non-linear Suspension System
}

\author{
A. G. Mohite, A. C. Mitra \\ (Department of Mechanical Engineering, M.E.S. College of Engineering, Pune, S.P. Pune University, India)
}

\begin{abstract}
In this paper development of Non-Linearvehicle suspension system iscarried out by anaylytically (State Space Equation) and MATLAB-SIMULINK model. The passive suspension has been studied with introducing Non-linearity approach to the system. A mathematical model is also developed for suspension system using Equilibrium of Motions. For the review purpose, a quarter car with two Degree of Freedom (DOF) model with presenting Non-linearity on stiffness and damping of a vehicle suspension framework have been produced. A MATLAB-SIMULINK model is created for Non-Linear vehicle suspension system to plot sprung mass displacement in time domain. The SIMULINK model is also validated with analytical model using State Space Equation and also a model with MATLAB-SIMULINK is developed to find RMS acceleration and settling time for Linear as well as Non-linear suspension system, which shows the considerable improvement of Nonlinear system over Linear, framework in configuration arrange as it only.
\end{abstract}

Keywords-Passive suspension systrm, Quarter car model, State space equation, MATLAB-SIMULINK.

\section{INTRODUCTION}

Suspension framework is one of the essential thought similar to vehicle security and solace to the traveler. The function of suspension system is to isolate the vehicle from various disturbances and irregularities so as to driver can keep control of the vehicle and also to carry weight of vehicle structure, driver, passenger and to absorb vibrations passing to the vehicle body [1]. As indicated by ISO 2631-1:1997 measures the estimation of the impact of vibration on the solace of a man in typical wellbeing who are presented to entire body occasional, irregular and transient vibration amid go, at work or amid recreation exercises are institutionalized are given by the Yufen Zhou, Suren Chen et.al. [1,2]. A. Mitra, N. Benerjee, et.al. are developed a MATLABSIMULINK model to examine the ride solace and vehicle taking care for various road profile Also the detail study of mathematical modelling with state space matrix are produced and approval of SIMULINK model with state space matrix are be finished [3]. Chen, Yinlong Hu et.al. explained thatthe passive suspension system is a conventional suspension system which contain two main components which are damper and spring. The execution of passive suspension system can be improved by utilizing semi active suspension system. The part of semi active suspension system is same as to passive suspension system difference is only that the external energy is needed in the system. The study of semi-active suspension by using the force tracking strategy is developed by the Chen and Yinlong Hu [4]. The linear spring are those one which have linear relationship between the force and displacement which means that the graph of force vs. displacement for linear spring is straight line, while in non-linear spring load vs. displacement graph are more complicated than straight line [5]. The Non-linearity's in damper can be explained by the graph of damping force vs. damper velocity is in complicated nature. The non-linearities are present in spring and damper due to reasons like the material properties, bump stop which confines the wheel go inside the given range and keeps the tire from reaching the vehicle body. Also, another is one that struct bushing which associates the struct with the body structure and lessens the cruelty from the street input [6]. M.P.Nagarkar, G.J. patil, etal outlined the Non-linear quarter auto suspension of seat-driver model and actualized for ideal plan, for Non-linear thought reason a quadratic tire firmness and cubic solidness in suspension spring, edge, and seat pad with 4 degrees of freedoms (DOF) driver model exhibited for advancement and investigation [7]. Lajqi.sh, pehan.s, developed analytical and experimental reviews on active and semi-active suspensions have been performed to enhance ride quality and handling execution [8]. A. mitra, Kiranchand.G.R., etal.explained design of experiment model of quarter car for optimization of automotive suspension system and find out ride comfort and road holding from regression model and experimentation [9].

In this paper passive suspension system has been considered with presenting Linearity and Nonlinearity way to deal with the system. A scientific model is produced utilizing State-Space equation and approved with MATLAB-SIMULINK model. The paper is composed as in first section quarter auto model for Linear and Non-Linear system is clarified. MATLAB program is generated for the analysis using state space model developed from the equations of motions. Program created here can be utilized for and vehicles quarter car show with 2-DOF for examination, which will be gainful in sparing cash required for test apparatuses and 
circuits. In the second section scientific modeling of Non-Linear suspension system is determined, and in section three Newton's $2^{\text {nd }}$ Law of motion is used to develop governing equation. In fourth and fifth section state space model and MATLAB-SIMULINK model are developed. In the sixth section results and discussion are explained. At last in seventh section conclusion are explained. In this study the effect of impediment as step function is explored for overshoot and settling time of sprung mass and concentrated the Non-linear conduct investigation. Also study compares the ride comfort and settling time qualities and its percentage variation with Linear and Non-linear model.

\section{QUARTER CAR MODEL}

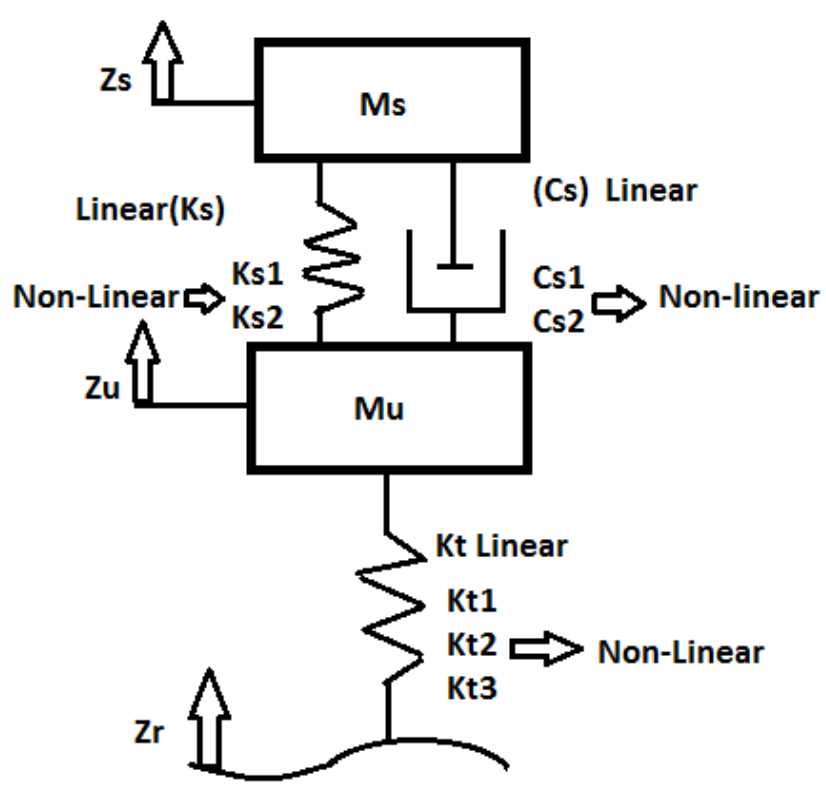

Fig.1. Quarter car model for passive suspension system

The vehicle model which studied for this paper is quarter car model. The quarter car model comprises of one-fourth of the aggregate body, as shown in Fig. 1. The quarter car model is set up using interconnections of masses, springs and dampers. In this diagram Ms sprung mass, Mu denotes un-sprung mass, Ks shows spring stiffness constant, Cs denotes dashpot constant and $\mathrm{Kt}$ is the spring constant of the tyre. $\mathrm{Zr}$ is the road or step input to the tyre, $\mathrm{Zs}$ and $\mathrm{Zu}$ shows the displacement acting at the mass Ms and Mu respectively. The lower springs and damper are portrayed by the tire stiffness for Linear system is $\mathrm{Kt}$ and for considering of Non-linear study varying the stiffness as $\mathrm{Kt} 1, \mathrm{Kt} 2, \mathrm{Kt} 3$, and the tire damping $\mathrm{Ct}$. Because of unimportant esteem disregard tire damping coefficient in our computation, and it have additionally immaterial impact on the last outcomes. The spring stiffness and damper damping coefficients are indicated by K for Linear system and K1, K2 for Nonlinear system and Cs for Linear system and Cs1, Cs2 for Non-linear suspension system. The un-sprung mass is empowered by the ground surface data $\mathrm{Zr}$ through the tires' contact. Forces that acting inside suspension systems can be described by independently as Linear and Non-linear characteristics are as shown in Fig.1.Below, the mathematical models for passive suspension systems are described. It is seen that the actual behavior of suspension system is better understands using the Non-linear attributes than Linear characteristics.

\section{MATHEMATICAL MODELING}

The conditions of movements for Linear framework can be composed as,

$$
\begin{aligned}
& M_{s} \ddot{Z}_{s}=-K_{s} \cdot\left(Z_{s}-Z_{u}\right)-C_{s} \cdot\left(\dot{Z}_{s}-\dot{Z}_{u}\right)(1) \\
& M_{u} \ddot{Z}_{u}=K_{s} \cdot\left(Z_{s}-Z_{u}\right)+C_{s} \cdot\left(\dot{Z}_{s}-\dot{Z}_{u}\right)-K_{t}\left(Z_{u}-Z_{r}\right)
\end{aligned}
$$

Non-linear behavioral strengths that demonstration in suspension is dictated by the accompanying expressions

$F_{t}=K_{t 1} \cdot\left(Z_{u}-Z_{r}\right)+K_{t 2} \cdot\left(Z_{u}-Z_{r}\right)^{2}-K_{t 3} \cdot\left(Z_{u}-Z_{r}\right)^{3}$

$F_{s}=K_{s 1} \cdot\left(Z_{s}-Z_{u}\right)+K_{s 2} \cdot\left(Z_{s}-Z_{u}\right)^{3}$

$F_{d}=C_{s 1} \cdot\left(\dot{Z}_{s}-\dot{Z}_{u}\right)+C_{s 2} \cdot\left(\dot{Z}_{s}-\dot{Z}_{u}\right)^{2}$ 
Equilibrium equations of motion for Non-linear suspension systems are,

$$
\begin{aligned}
& M_{s} \cdot \ddot{Z}_{s}=-K_{s 1} \cdot\left(Z_{s}-Z_{u}\right)-K_{s 2} \cdot\left(Z_{s}-Z_{u}\right)^{3}-C_{s 1} \cdot\left(\dot{Z}_{s}-\dot{Z}_{u}\right)-C_{s 2} \cdot\left(\dot{Z}_{s}-\dot{Z}_{u}\right)^{2} \\
& M_{u} \cdot \ddot{Z}_{u}=K_{s 1} \cdot\left(Z_{s}-Z_{u}\right)+K_{s 2} \cdot\left(Z_{s}-Z_{u}\right)^{3}+C_{s 1} \cdot\left(\dot{Z}_{s}-\dot{Z}_{u}\right)+C_{s 2} \cdot\left(\dot{Z}_{s}-\dot{Z}_{u}\right)^{2}-K_{t 1} \cdot\left(Z_{u}-Z_{r}\right)-K_{t 2} \cdot\left(Z_{u}-Z_{r}\right)^{2}+K_{t 3} \cdot\left(Z_{u}-Z_{r}\right)^{3}
\end{aligned}
$$

Where $\ddot{Z}_{s}, \dot{Z}_{s}, Z_{s}$ and $\ddot{Z}_{u}, \dot{Z}_{u}, Z_{u}$ signify the acceleration, velocity, and displacement of the sprung and unsprung masses, separately. Equations (1), (2), (3), and (4) shows equilibrium equation of motions for Linear and Non-linear passive suspension systems, individually.Suspension parameter are taken for linear suspension system are $K_{s}=9015 \mathrm{~N} / \mathrm{m}, C_{s}=2031 \mathrm{Ns} / \mathrm{m}, K_{t}=41815 \mathrm{~N} / \mathrm{m}, M_{s}=295 \mathrm{Kg}$, and $M_{u}=39 \mathrm{Kg}$. The sprung mass acceleration for Linear suspension system are plotted out and shown in fig. 4.

\section{Non-linear suspension Parameters for quarter car model.}

Table1- Non-linear Suspension Parameter

\begin{tabular}{|l|l|l|l|}
\hline Suspension parameters & Symbol & Unit & Value \\
\hline Sprung mass & $M_{s}$ & $K g$ & 295 \\
\hline Un-sprung mass & $M_{u}$ & $K g$ & 39 \\
\hline Linear damping coefficient & $C_{s 1}$ & $N s / m$ & 3482 \\
\hline Non-linear square damping coefficient & $C_{s 2}$ & $N s / m^{2}$ & 580 \\
\hline Linear spring stiffness coefficient & $K_{s 1}$ & $N / m$ & 15302 \\
\hline Non-linear square spring stiffnesscoefficient & $K_{s 2}$ & $N / m^{3}$ & 2728 \\
\hline Linear tire stiffness coefficient & $K_{t 1}$ & $N / m$ & 60063 \\
\hline Non-linear square tire stiffnesscoefficient & $K_{t 2}$ & $N / m^{2}$ & 42509 \\
\hline Non-linear cube tire stiffnesscoefficient & $K_{t 3}$ & $N / m^{3}$ & 22875 \\
\hline
\end{tabular}

\section{ANALYTICAL SOLUTION (STATE SPACE MODEL) FOR NON-LINEAR SUSPENSION} SYSTEM

With a specific end goal to explain the differential conditions of movement for the general case of ground excitation, state space model is very quickly and effective technique to accelerate the numerical methodology. Numerical simulations have been created for all spoke to suspension systems shown in fig.1. Then Eq. (5) and (6) is changed into the state variable equations by rearranging the second-arrange differential conditions changed over into first-arrange differential conditions. To minimize difficulties in calculation higher order degree terms are eliminated in equation (5) and (6), by eliminating higher order degree terms equation (5) and (6) changes to,

$$
\begin{aligned}
& M_{s} \cdot \ddot{Z}_{s}=-K_{s 1} \cdot\left(Z_{s}-Z_{u}\right)-K_{s 2} \cdot\left(Z_{s}-Z_{u}\right)-C_{s 1} \cdot\left(\dot{Z}_{s}-\dot{Z}_{u}\right)-C_{s 2} \cdot\left(\dot{Z}_{s}-\dot{Z}_{u}\right) \\
& M_{u} \cdot \ddot{Z}_{u}=K_{s 1} \cdot\left(Z_{s}-Z_{u}\right)+K_{s 2} \cdot\left(Z_{s}-Z_{u}\right)+C_{s 1} \cdot\left(\dot{Z}_{s}-\dot{Z}_{u}\right)+C_{s 2} \cdot\left(\dot{Z}_{s}-\dot{Z}_{u}\right)-K_{t 1} \cdot\left(Z_{u}-Z_{r}\right)-K_{t 2} \cdot\left(Z_{u}-Z_{r}\right)+K_{t 3} \cdot\left(Z_{u}-Z_{r}\right)
\end{aligned}
$$
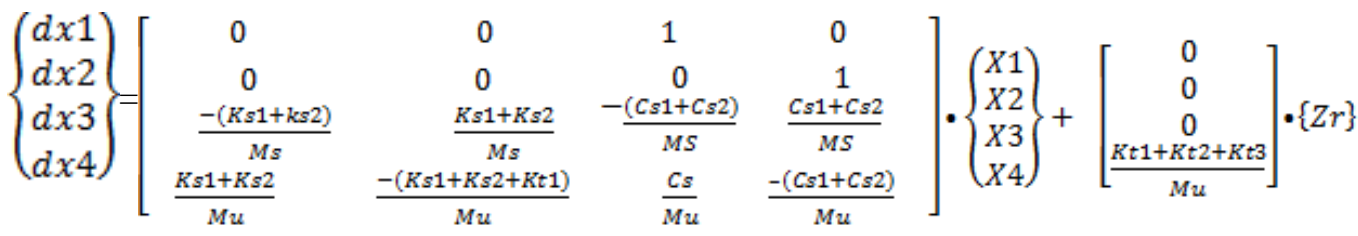

These are the state space equations are in the form of,

$$
\begin{aligned}
& \dot{X}=A x+B u \\
& Y=C x+D u
\end{aligned}
$$


Where A matrix appears as state matrix, B is information matrix, $\mathrm{C}$ is the yield matrix and D is the immediate transmission matrix.

\section{MATLAB-SIMULINK FOR NON-LINEAR SUSPENSION SYSTEM}

From equations (5) and (6), mathematical model of passive Non-linear system is made in MATLABSIMULINK shown in fig. (2). Suspension parameter values are taken from the Table1.The purpose of this to plot sprung mass displacement in time domain.

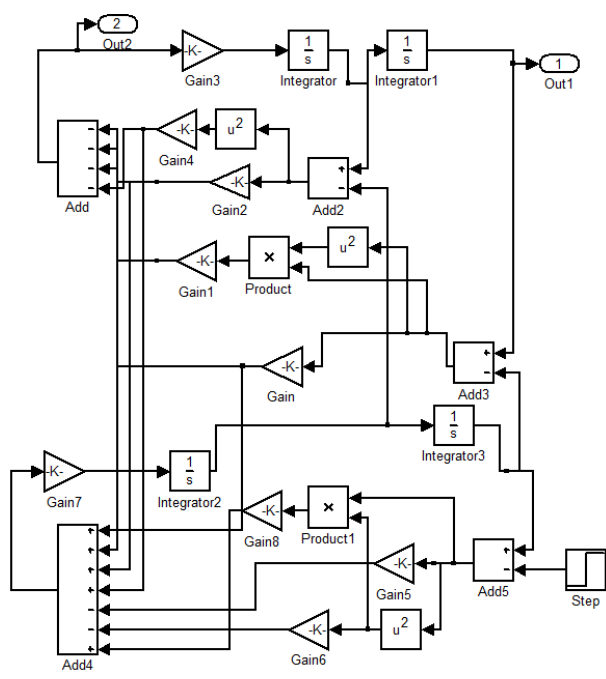

Fig.2 SIMULINK model of Non-linear vehicle suspension system.

\section{Validation of Non-linear suspension system}

\section{RESULTS AND DISCUSSION}

The validation ofSprung mass displacement $\left(Z_{s}\right)$. Vs. time is as shown in fig.3. the sprung mass displacement obtained from Analytival solution is at left side and while sprung mass displacement obtained by MATLAB-SIMULINK model is at right side in Fig.3. By analysis it is seen that the nature of the graph is quite similar to each other. A small difference is occurred due to the higher order degree terms are eliminated in the state space equation to minimize the difficulties in calculation. Graph obtained by state space equation. (Analytical solution).


Graph obtained by MATLAB simulation.

Fig.3 Validation Graph of sprung mass displacement of Non-linear system 
Sprung mass acceleration for Linear and Non-linear vehicle suspension system.



Fig.4 sprung mass displacement of both Linear and Non-linear system

RMS acceleration value and settling time value.

Table2-Results of RMS acceleration values and settling time

\begin{tabular}{|l|l|l|l|l|}
\hline System & RMS acceleration value in $\mathrm{m} / \mathrm{s}^{2}$ & \%variation & Settling time in Sec & \%variation \\
\hline Linearsuspension system & 0.1238 & \multirow{2}{*}{35.48} & 1.2 & \multirow{2}{*}{-4.35} \\
\cline { 1 - 2 } $\begin{array}{l}\text { Non-linearsuspension } \\
\text { system }\end{array}$ & 0.1919 & 1.15 & \\
\hline
\end{tabular}

The Fig.4.shows sprung mass acceleration $\left(\ddot{Z}_{s}\right)$ vs. time graph for linear as well as Non-linear suspension syatem. There is considerable difference in both the system. The RMS accelratiom value for linear suspension system is 0.1238 while RMS acceleration value for Non-linear suspension system is 0.1919 , so \%variation is 35.48 for RMS acceleration value. While settling time is 1.2 and $1.15 \mathrm{sec}$ respectively for linear and Non-linear system.

\section{CONCLUSION}

It is seen that, to study the Non-linear behavior of suspension system is important and it is necessary to consider the Non-linearity's in suspension system for analysis of dynamic vehicle suspension system. It is found that the disorderly reaction exists in Non-linear suspension. As the results of analytical (State Space Equation) and MATLAB-SIMULINK analysis of quarter car passive Non-linear suspension system are quite similar. Small difference is found in nature of graph because of higher order degree terms are eliminated in calculation of state space equation to avoid difficulties in calculation. The RMS value of sprung mass acceleration for Linear suspension system is $0.1238 \mathrm{~m} / \mathrm{s}^{2}$ while in Non-linear suspension system RMS acceleration value for sprung mass is increases and it goes to $0.1919 \mathrm{~m} / \mathrm{s}^{2}$. The percentage variation for RMS acceleration value is $35.48 \%$. settling time Linear And Non-linear suspension system is 1.2 and 1.15 respectively, and percentage variation is $-4.35 \%$. Consequently it is infer that by considering Non-linear way to deal with the system then systematic outcomes are goes closer to the genuine one, in configuration organize as it were.

\section{REFERENCES}

[1] T.P. Phalake, A.C. Mitra, Design and Analysis of vehicle suspension system,International Engineering Research Journal,165-172.

[2] Y. Zhou, S. Chen.,Vehicle ride comfort analysis with whole-body vibration on long-span bridges subjected to crosswind, Journal of Wind Engineering and Industrial Aerodynamics. J. WindEng.Ind.Aerodyn.155,2016,126-140.

[3] A. Mitra, N. Benerjee, H. A. Khalane, M. A. Sonawane, D. R. Joshi, G.R. Bagul,Simulation and Analysis of Full Car Model for various Road profile on a analytically validated MATLAB/SIMULINK model,IOSR Journal of Mechanical and Civil Engineering (IOSR-JMCE) ISSN(e) : 2278-1684, ISSN(p) : 2320-334X, PP : 22-33. 
[4] M. Z. Q. Chen, Y.Hu, C. Li Guanrong Chen,Semi-active suspension with semi-active inerter and semi-active damper, Proceedings of the 19th World Congress The International Federation of Automatic Control Cape Town, South Africa, August 24-29, 2014

[5] R. kerumali, S.H. Sawant,Analysis of Non-linearity in spring and damper of vibration isolator subjected to harmonic excitation, SSRG International journal of mechanical engineering,1( 3),2014,5-9.

[6] S. P. Chavan, S.H.Sawant,Experimental verification of passive half car vehicle dynamics system subjected to harmonic road excitation with Non-linear parameters, International Conference on Challenges and Opportunities in Mechanical Engineering, Industrial Engineering and Management Studies, (ICCOMIM - 2012), 2012,527-532.

[7] M.P. Nagarkar ,G. J. VikhePatil, Rahul N. Z..Patil,Optimization of nonlinear quarter car suspension-seat-driver model, Journal of Advanced Research, 7(6),2016, 991-1007.

[8] A. C. Mitra,G.R.Kiranchand, Tanushri Soni, N.Banerjee., Design of Experiments For Optimization of Automotive Suspension System Using Quarter Car Test Rig, Procedia Engineering,, International Conference on Vibration Problems 2015,144,2016,1102-1109.

[9] S. Lajqi,S.Pehan, pehan,N.Lajqi,A.Gjelag,S.Emin, Design of independent suspension mechanism for a terrain vehicle with four wheels drive and four wheels steering,International Journal of Engineering,(2013) MOTSP conference proceedings, 2013,230-237. 